\title{
The scaling window for a random graph with a given degree sequence
}

\author{
Hamed Hatami and Michael Molloy* \\ Department of Computer Science \\ University of Toronto \\ e-mail: hamed@cs.toronto.edu, molloy@cs.toronto.edu
}

\begin{abstract}
We consider a random graph on a given degree sequence $\mathcal{D}$, satisfying certain conditions. We focus on two parameters $Q=Q(\mathcal{D}), R=R(\mathcal{D})$. Molloy and Reed proved that $Q=0$ is the threshold for the random graph to have a giant component. We prove that if $|Q|=O\left(n^{-1 / 3} R^{2 / 3}\right)$ then, with high probability, the size of the largest component of the random graph will be of order $\Theta\left(n^{2 / 3} R^{-1 / 3}\right)$. If $|Q|$ is asymptotically larger than $n^{-1 / 3} R^{2 / 3}$ then the size of the largest component is asymptotically smaller or larger than $n^{2 / 3} R^{-1 / 3}$. Thus, we establish that the scaling window is $|Q|=O\left(n^{-1 / 3} R^{2 / 3}\right)$.
\end{abstract}

\section{Introduction}

The double-jump threshold, discovered by Erdös and Rényi[9], is one of the most fundamental phenomena in the theory of random graphs. The component structure of the random graph $G_{n, p=c / n}$ changes suddenly when $c$ moves from below one to above one. For every constant $c<1$, almost surely ${ }^{1}$ (a.s.) every component has size $O(\log n)$, at $c=1$ a.s. the largest component has size of order $\Theta\left(n^{2 / 3}\right)$, and at $c>1$ a.s. there exists a single giant component of size $\Theta(n)$ and all other components have size $O(\log n)$. For this reason, $c=1$ is often referred to as the critical point.

In the 1980's, Bollobás[5], Euczak[16] and others studied the case where $p=\frac{1+o(1)}{n}$. They showed that when $p=\frac{1}{n}+\frac{c}{n^{1 / 3}}$ for any constant $c$ (positive or negative), the component sizes of $G_{n, p}$ behave as described above for $p=\frac{1}{n}$. Furthermore, if $p$ lies outside of that range, then the size of the largest component behaves very differently: For larger/smaller values of $p$, a.s. the largest component has size asymptotically larger/smaller than $\Theta\left(n^{2 / 3}\right)$. That range of $p$ is generally referred to as the scaling window. See, eg. [6] for further details.

Molloy and Reed[17] proved that something analogous to the cases $c<1$ and $c>1$ holds for random graphs on a given degree sequence. They considered a sequence $\mathcal{D}=\left(d_{1}, \ldots, d_{n}\right)$ satisfying certain conditions, and chose a graph uniformly at random from amongst all graphs with that degree sequence. They determined a parameter $Q=Q(\mathcal{D})$ such that if $Q<0$ then a.s. every component has size $O\left(n^{x}\right)$ for some $x<1$ and if $Q>0$ then a.s. there exists a single giant component of size $\Theta(n)$ and all other components have size $O(\log n)$.

In this paper, we establish a scaling window around the threshold $Q=0$, under certain conditions for $\mathcal{D}$. We will state our results more formally in the next subsection, but in short: If $\sum d_{i}^{3}=O(n)$, then the situation is very much like that for $G_{n, p}$. The scaling window is the range $|Q|=O\left(n^{-1 / 3}\right)$

\footnotetext{
${ }^{*}$ Research supported by an NSERC Discovery Grant.

${ }^{1} \mathrm{~A}$ property $\mathrm{P}$ holds almost surely if $\lim _{n \rightarrow \infty} \operatorname{Pr}(P)=1$.
} 
and inside the scaling window, the size of the largest component is $\Theta\left(n^{2 / 3}\right)$. As discussed below, the conditions required in $[13,12]$ imply that $\sum d_{i}^{3}=O(n)$, which explains why they obtained their results. If $\sum d_{i}^{3} \gg n$, then the situation changes: the size of the scaling window becomes asympotically larger, and the size of the largest component becomes asymptotically smaller.

\subsection{The main results}

Before stating our theorems, we will introduce some notation:

We are given a set of vertices along with the degree $d_{v}$ of each vertex. We denote this degree sequence by $\mathcal{D}$. We assume that there is at least one graph with degree sequence $\mathcal{D}$ (and so, eg., $\sum_{v} d_{v}$ is even). Our random graph is selected uniformly from amongst all graphs with degree sequence $\mathcal{D}$.

We use $E$ to denote the set of edges, and note that $|E|=\frac{1}{2} \sum_{v \in G} d_{v}$. We let $n_{i}$ denote the number of vertices of degree $i$. We use $\mathcal{C}_{\max }$ to denote the largest component of the random graph. We define:

$$
\begin{gathered}
Q:=Q(\mathcal{D}):=\frac{\sum_{u \in G} d_{u}^{2}}{2|E|}-2, \\
R:=R(\mathcal{D}):=\frac{\sum_{u \in G} d_{u}\left(d_{u}-2\right)^{2}}{2|E|} .
\end{gathered}
$$

The relevance of $Q, R$ will be made clear in Section 2.4. The asymptotic order of $R$ is important; note that, when $|E| / n$ and $Q$ are bounded by constants, $R$ has the same order as $\frac{1}{n} \sum_{u \in G} d_{u}^{3}$. The order of $R$ was implicitly seen to be important in the related papers $[12,13]$, where they required $\frac{1}{n} \sum_{u \in G} d_{u}^{3}$ to be bounded by a constant (see Section 1.3).

Molloy and Reed [17] proved that, under certain assumptions about $\mathcal{D}$, if $Q$ is at least a positive constant, then a.s. $\left|\mathcal{C}_{\max }\right| \geq c n$ for some $c>0$ and if $Q$ is at most a negative constant then a.s. $\left|\mathcal{C}_{\max }\right| \leq n^{x}$ for some constant $x<1$. Some of these assumptions were that the degree sequence converged in certain ways as $n \rightarrow \infty$; in particular, $n_{i} / n$ converged to a limit for all $i$ uniformly, and $Q$ converged to $\frac{\sum_{i>0} i^{2} \times \lim _{n \rightarrow \infty} n_{i} / n}{2|E|}-2$. We don't require those assumptions in this paper.

But we do require some assumptions about our degree sequence. First, it will be convenient to assume that every vertex has degree at least one. A random graph with degree sequence $d_{1}, \ldots, d_{n}$ where $d_{i}=0$ for every $i>n^{\prime}$ has the same distribution as a random graph with degree sequence $d_{1}, \ldots, d_{n^{\prime}}$ with $n-n^{\prime}$ vertices of degree zero added to it. So it is straightforward to apply our results to degree sequences with vertices of degree zero.

Anomalies can arise when $n_{2}=n-o(n)$. For example, in the extreme case where $n_{2}=n$, we have a random 2-regular graph, and in this case the largest component is known to have size $\Theta(n)$ (see eg. [2]). So we require that $n_{2} \leq(1-\zeta) n$ for some constant $\zeta>0$. [12, 13] required that $n_{1}>\zeta n$ - note that requirement is equivalent to ours when $n_{0}=0$ and $Q=o(1)$. See Remark 2.7 of [12] for a description of some other behaviours that can arise when we allow $n_{2}=n-o(n)$.

As in $[17,18]$ and most related papers (eg. $[10,12,13]$ ), we require an upper bound on the maximum degree, $\Delta$. We take $\Delta \leq n^{1 / 3} R^{1 / 3}(\ln n)^{-1}$, which is higher than the bounds from $[12,13,17,18]$ and is nearly as high as $\Delta$ can possibly be in this setting (see Section 1.2).

Finally, since we are concerned with $Q=o(1)$, we can assume $|Q| \leq \frac{\zeta}{2}$, and that $\zeta$ is sufficiently small, eg. $\zeta<\frac{1}{10}$. In summary, we assume that $\mathcal{D}$ satisfies the following:

Condition D: For some constant $0<\zeta<\frac{1}{10}$

(a) $\Delta \leq n^{1 / 3} R^{1 / 3}(\ln n)^{-1}$;

(b) $n_{0}=0$; 
(c) $n_{2} \leq(1-\zeta) n ;$

(d) $|Q| \leq \frac{\zeta}{2}$

Our main theorems are:

Theorem 1.1 For any $\lambda, \epsilon, \zeta>0$ there exist $A, B$ and $N$ such that for any $n \geq N$ and any degree sequence $\mathcal{D}$ satisfying Condition $D$ and with $-\lambda n^{-1 / 3} R^{2 / 3} \leq Q \leq \lambda n^{-1 / 3} R^{2 / 3}$, we have

(a) $\operatorname{Pr}\left[\left|\mathcal{C}_{\max }\right| \leq A n^{2 / 3} R^{-1 / 3}\right] \leq \epsilon$;

(b) $\operatorname{Pr}\left[\left|\mathcal{C}_{\max }\right| \geq B n^{2 / 3} R^{-1 / 3}\right] \leq \epsilon$.

Theorem 1.2 For any $\epsilon, \zeta>0$ and any function $\omega(n)$ tending to $\infty$ with $n$, there exists $B, N$ such that for any $n \geq N$ and any degree sequence $\mathcal{D}$ satisfying Condition $D$ and with $Q<-\omega(n) n^{-1 / 3} R^{2 / 3}$ we have:

(a) $\operatorname{Pr}\left(\left|\mathcal{C}_{\max }\right| \geq B \sqrt{n /|Q|}\right)<\epsilon$.

(b) The probability that the random graph contains a component with more than one cycle is at most $\frac{20}{\omega(n)^{3}}$.

Theorem 1.3 For any $\epsilon, \zeta>0$ and any function $\omega(n)$ tending to $\infty$ with $n$, there exists $A, N$ such that for any $n \geq N$ and any degree sequence $\mathcal{D}$ satisfying Condition $D$ and with $Q>\omega(n) n^{-1 / 3} R^{2 / 3}$ :

$$
\operatorname{Pr}\left(\left|\mathcal{C}_{\max }\right| \leq A Q n / R\right)<\epsilon .
$$

Note that the bounds on $\left|\mathcal{C}_{\max }\right|$ in Theorems 1.2 and 1.3 are $B \sqrt{n /|Q|}<B n^{-1 / 3} R^{2 / 3} / \sqrt{\omega(n)}$ and $A Q n / R>A \omega(n) n^{2 / 3} R^{-1 / 3}$. So our theorems imply that $|Q|=O\left(n^{-1 / 3} R^{2 / 3}\right)$ is the scaling window for any degree sequences that satisfy Condition $\mathrm{D}$, and that in the scaling window the size of the largest component is $\Theta\left(n^{2 / 3} R^{-1 / 3}\right)$.

Note also that Theorem 1.2(b) establishes that when $Q$ is below the scaling window then, with high probability, every component is either a tree or is unicyclic. This was previously known to be the case for the $G_{n, p}$ model[16].

The approach we take for Theorems 1.1 and 1.3 closely follows that of Nachmias and Peres[19] who applied some Martingale analysis, including the Optional Stopping Theorem, to obtain a short elegant proof of what happens inside the scaling window for $G_{n, p=c / n}$. See also [20] where they apply similar analysis to also obtain a short proof of what happens outside the scaling window, including tight bounds on the size of the largest component.

The approach we take for Theorem 1.2 is a first moment argument similar in spirit to one applied in [16] to $G_{n, p}$, along with a very simple Martingale analysis.

\subsection{Our bound on $\Delta$}

Since there is a vertex $v$ of degree $\Delta$, we always have $R>\frac{d_{v}\left(d_{v}-2\right)^{2}}{2|E|} \geq \frac{\Delta^{3}}{18|E|}$. Lemma 2.1 in the next section gives $|E| \leq\left(1+\frac{1}{2} Q\right) n<2 n$. This yields $R>\frac{\Delta^{3}}{36 n}$ and hence $\Delta<4 n^{1 / 3} R^{1 / 3}$. So our bound on $\Delta$ is within a factor of $O(\log n)$ of the maximum that $\Delta$ can possibly be when $|Q|=o(1)$. In fact, it is possible to reduce this factor somewhat - our arguments still work if $\Delta \leq \kappa n^{1 / 3} R^{1 / 3}(\log n)^{-1 / 2}$ for some sufficiently small constant $\kappa$ that depends on $\lambda, \epsilon, \zeta$. But it can't be eliminated entirely:

Consider a degree sequence where one vertex $v$ has degree $\Delta \gg n^{1 / 3}$, all other vertices have small degree and the contribution of those other vertices to $Q$ is $O\left(n^{-1 / 3}\right)$; eg. $\frac{3}{4}$ of them have degree 1 
and the others have degree 3. Then we have $R=\Theta\left(\frac{\Delta^{3}}{n}\right)$ and $Q=\Theta\left(\frac{\Delta^{2}}{n}\right)=\Theta\left(n^{-1 / 3} R^{2 / 3}\right)$ and so it is within what our results say is the scaling window. However, the same arguments that we use to prove Theorem 1.1(a) will prove that, with high probability, the random graph has a component of size $\Theta\left(n^{2 / 3}\right) \gg n^{2 / 3} R^{-1 / 3}$ (see the remark following that proof in Section 6).

What causes that degree sequence to behave in this manner is that $R$ is large entirely because of a single vertex. If we remove $v$, then the remaining degree sequence has $R=O(1)$. Note that our bound on $\Delta$ in Condition $\mathrm{D}$ is equivalent to $R>\frac{\Delta^{3}(\ln n)^{3}}{n}$ and so it is always satisfied if, eg., there are at least $(\ln n)^{3}$ vertices of degree $\Delta$. Our bound on $\Delta$ can be viewed as a condition that the asymptotic order of $R$ is determined by several high degree vertices. On the other hand, there are counterexamples when it is determined by a small number of vertices.

\subsection{Related Work}

In 2000, Aiello, Chung and Lu[1] applied the results of Molloy and Reed[17, 18] to a model for massive networks. They also extended those results to apply to power law degree sequences with maximum degree higher than that required by $[17,18]$. Since then, that work been used numerous times to analyze massive network models arising in a wide variety of fields such as physics, sociology and biology (see eg. [21]).

Cooper and Frieze[8] proved, amongst other things, an analogue of the main results of $[17,18]$ in the setting of giant strongly connected components in random digraphs.

Fountoulakis and Reed[10] extended the work of [17] to degree sequences that do not satisfy the convergence conditions required by [17]. They require $\Delta \leq|E|^{1 / 2-\epsilon}$ which in their setting implies $\Delta \leq O\left(n^{1 / 2-\epsilon}\right)$.

Kang and Seierstad[13] applied generating functions to study the case where $Q=o(1)$, but is outside of the scaling window. They require a maximum degree of at most $n^{1 / 4-\epsilon}$ and that the degree sequences satisfy certain conditions that are stronger than those in [17]; one of these conditions implies that $R$ is bounded by a constant. Based on what is known for $G_{n, p}$, it was natural to guess that for $|Q| \gg n^{-1 / 3}$ we would have $\left|\mathcal{C}_{\max }\right| \neq \Theta\left(n^{2 / 3}\right)$. They proved that if $Q \ll-n^{-1 / 3}$ then $\left|\mathcal{C}_{\max }\right| \ll n^{2 / 3}$, and if $Q \gg n^{-1 / 3} \log n$ then $\left|\mathcal{C}_{\max }\right| \gg n^{2 / 3}$. So for the case where $R=O(1)$ is bounded, this almost confirmed that natural guess - except that they did not cover the range where $n^{-1 / 3} \ll Q=O\left(n^{-1 / 3} \log n\right)$.

Jansen and Luczak[12] used simpler techniques to obtain a result along the lines of that in [13]. They require a maximum degree of $n^{1 / 4}$, and they also require $R=O(1)$; in fact, they require $\frac{1}{n} \sum_{v} d_{v}^{4+\eta}$ to be bounded by a constant (for some arbitrarily small constant $\eta>0$ ), but they conjecture that having $\frac{1}{n} \sum_{v} d_{v}^{3}$ bounded (i.e. $R$ bounded) would suffice. For $Q \gg n^{-1 / 3}$, they prove that $\left|\mathcal{C}_{\max }\right|=$ $\Theta(n Q) \gg n^{2 / 3}$. Thus (in the case that their conditions hold) they eliminated the gap left over from [13]. Furthermore, this also shows that the asymptotic order of $\left|\mathcal{C}_{\text {max }}\right|$ increases with $|Q|$ in that range, thus eliminating the possibility of a scaling window extending into that range. They also used their techniques to obtain a simpler proof of the main results from [17, 18].

So for the case $R=O(1)$, the bound on the scaling window provided by Theorems $1.2(\mathrm{a})$ and 1.3 was previously known (under somewhat stronger conditions). But it was not known that $|Q|=O\left(n^{-1 / 3}\right)$ was indeed the scaling window; it was possibly smaller. In fact, it was not even clear that there was any scaling window in terms of $Q$ at all. No bounds on $\left|\mathcal{C}_{\max }\right|$ were known for when $|Q|=O\left(n^{-1 / 3}\right)$. And nothing was known for the case when $R$ grows with $n$. 


\section{Preliminaries}

\section{$2.1 \quad$ Some Observations}

We start with two easy observations.

Lemma 2.1 If $\mathcal{D}$ satisfies Condition $D(b, c)$ then:

(a) $\frac{1}{2} n \leq|E| \leq\left(1+\frac{1}{2} Q\right) n$, and $\sum_{u \in V} d_{u}^{2}=(4+2 Q)|E|$.

(b) $\frac{\zeta}{4} \leq R \leq 2 \Delta$.

Proof.

Part (a)

$$
2|E| Q=-4|E|+\sum_{u \in V} d_{u}^{2},
$$

which establishes the second assertion. Now by the Cauchy-Schwarz inequality,

$$
2|E|=\sum_{u \in V} d_{u} \leq \sqrt{n} \sqrt{\sum_{u \in V} d_{u}^{2}} \leq \sqrt{n} \sqrt{4|E|\left(1+\frac{1}{2} Q\right)},
$$

which shows that $|E| \leq\left(1+\frac{1}{2} Q\right) n$. The fact that every vertex has degree at least one implies $|E| \geq \frac{1}{2} n$.

Part (b)

$$
R=\sum_{u \in V} \frac{d_{u}\left(d_{u}-2\right)^{2}}{2|E|} \geq \sum_{u: d_{u} \neq 2} \frac{d_{u}}{2|E|} \geq \frac{\zeta n}{2|E|} \geq \frac{\zeta}{4}
$$

On the other hand for sufficiently large $n$,

$$
\begin{aligned}
R & =\sum_{u} \frac{d_{u}\left(d_{u}-2\right)^{2}}{2|E|} \leq(\Delta-2)\left(\sum_{u: d_{u}>1} \frac{d_{u}\left(d_{u}-2\right)}{2|E|}\right)+\frac{n_{1}}{2|E|} \\
& \leq(\Delta-2)\left(Q+\frac{n_{1}}{2|E|}\right)+\frac{n_{1}}{2|E|} \leq(\Delta-2)(Q+1)+1<2 \Delta
\end{aligned}
$$

since $|Q|<1$.

\subsection{The Random Model}

In order to generate a random graph with a given degree sequence $\mathcal{D}$, we use the configuration model due to Bollobás[4] and inspired by Bender and Canfield[3]. In particular, we:

- Form a set $L$ which contains $d_{v}$ distinct copies of every vertex $v$.

- Choose a random perfect matching over the elements of $L$.

- Contract the different copies of each vertex $v$ in $L$ into a single vertex.

This may result in a graph $\mathcal{G}(\mathcal{D})$ with multiple edges and loops, but our conditions on $\mathcal{D}$ imply that for $n$ sufficiently large, the probability that $\mathcal{G}(\mathcal{D})$ is simple is bounded away from zero. Furthermore if one conditions on $\mathcal{G}(\mathcal{D})$ being simple, then it is uniformly distributed over the simple graphs with degree sequence $\mathcal{D}$. This allows us to translate results about $\mathcal{G}(\mathcal{D})$ to results about a uniform simple graph with degree sequence $\mathcal{D}$. 
Proposition 2.2 Consider any degree sequence $\mathcal{D}$ satisfying Condition $D(b, c, d)$. Suppose that a property $\mathcal{P}$ holds with probability at most $\epsilon$ for a uniformly random configuration with degree sequence $\mathcal{D}$. Then for a uniformly random graph with degree sequence $\mathcal{D}, \operatorname{Pr}(\mathcal{P}) \leq \epsilon \times e$.

Proof. Let $\mathcal{H}$ be a random configuration with degree sequence $\mathcal{D}$. Lemma 2.1(b) implies that $\Delta=o\left(|E|^{1 / 2}\right)$. This allows us to apply Corollary 1.5 of [11], which states that the probability of our configuration being simple is

$$
e^{\frac{1}{4}-\frac{1}{4}\left(\frac{\sum_{v \in G} d_{v}^{2}}{2|E|}\right)^{2}}+o(1)
$$

Lemma 2.1(a) implies that this is at most

$$
e^{\frac{1}{4}-\frac{1}{4}\left(\frac{4+2 Q}{2+Q}\right)^{2}}+o(1)=e^{-3 / 4}+o(1)>e^{-1},
$$

for $n$ sufficiently large. The probability that a random graph with degree sequence $\mathcal{D}$ has $\mathcal{P}$ is:

$$
\operatorname{Pr}(\mathcal{H} \text { has } \mathcal{P} \mid \mathcal{H} \text { is simple }) \leq \operatorname{Pr}(\mathcal{H} \text { has } \mathcal{P}) / \operatorname{Pr}(\mathcal{H} \text { is simple }) \leq \epsilon \times e .
$$

\subsection{Martingales}

A random sequence $X_{0}, X_{1}, \ldots$ is a martingale if for all $i \geq 0, \mathbb{E}\left(X_{i+1} \mid X_{0}, \ldots, X_{i}\right)=X_{i}$. It is a submartingale, resp. supermartingale, if for all $i \geq 0, \mathbb{E}\left(X_{i+1} \mid X_{0}, \ldots, X_{i}\right) \geq X_{i}$, resp. $\mathbb{E}\left(X_{i+1} \mid X_{0}, \ldots, X_{i}\right) \leq X_{i}$.

A stopping time for a random sequence $X_{0}, X_{1}, \ldots$ is a step $\tau$ (possibly $\tau=\infty$ ) such that we can determine whether $i=\tau$ by examining only $X_{0}, \ldots, X_{i}$. It is often useful to view a sequence as, in some sense, halting at time $\tau$; a convenient way to do so is to consider the sequence $X_{\min (i, \tau)}$, whose $i$ th term is $X_{i}$ if $i \leq \tau$ and $X_{\tau}$ otherwise.

In our paper, we will make heavy use of the Optional Stopping Theorem. The version that we will use is the following, which is implied by Theorem 17.6 of [15]:

The Optional Stopping Theorem Let $X_{0}, X_{1}, \ldots$ be a martingale (resp. submartingale, supermartingale), and let $\tau \geq 0$ be a stopping time. If there is a fixed bound $T$ such that $\operatorname{Pr}(\tau \leq T)=1$ then $\mathbb{E}\left(X_{\tau}\right)=X_{0}$ (resp. $\left.\mathbb{E}\left(X_{\tau}\right) \geq X_{0}, \mathbb{E}\left(X_{\tau}\right) \leq X_{0}\right)$. $[7]$.

We will also use the following concentration theorem, which is given by Theorems 6.1 and 6.5 from

Theorem 2.3 Let $X_{0}, X_{1}, \ldots$ be a martingale satisfying

(a) $\operatorname{Var}\left(X_{i} \mid X_{0}, X_{1}, \ldots, X_{i-1}\right) \leq \sigma_{i}^{2}$, for $1 \leq i \leq n$.

(b) $\left|X_{i}-X_{i-1}\right| \leq M$, for $1 \leq i \leq n$.

Then

$$
\operatorname{Pr}[|X-\mathbb{E} X| \geq \rho] \leq 2 e^{-\frac{\rho^{2}}{2\left(M \rho+\sum \sigma_{i}^{2}\right)}}
$$

\subsection{The Branching Process}

As in [17], we will examine our random graph using a branching process of the type first applied to random graphs by Karp in [14]. This time, we need to be much more careful, since the branching parameter is $Q+1$ which can be $1+o(1)$. 
Given a vertex $v$, we explore the graph $\mathcal{G}(\mathcal{D})$ starting from $v$ in the following manner. At step $t$, we will have a partial subgraph $C_{t}$ which has been exposed so far. Typically, there will be some vertices of $C_{t}$ whose neighbours have not all been exposed; since we are working in the configuration model, this is equivalent to saying that there are some vertex-copies of vertices in $C_{t}$ whose partners in the configuration have not been exposed. We choose one of those vertex-copies and expose its partner by selecting that partner uniformly at random from amongst all vertex-copies that are still unmatched; if the partner is a vertex-copy of a vertex $u \notin C_{t}$, then we add $u$ to $C_{t}$. This yields an edge of $C_{t}$. If all the vertex-copies of all vertices in $C_{t}$ are matched, then this indicates that we have exposed an entire component. So we start exploring a new component beginning with an arbitrary vertex. Note that $C_{t}$ may contain several components, but that all vertices with unmatched vertex-copies belong to the same component - the one that is currently being explored.

We will use $Y_{t}$ to denote the total number of unmatched vertex-copies of vertices in $C_{t}$. So $Y_{t}=0$ indicates that we have exposed an entire component and are about to start a new one.

1. Choose an arbitrary vertex $v$ and initialize $C_{0}=\{v\} ; Y_{0}=\operatorname{deg}(v)$.

2. Repeat while there are any vertices not in $C_{t}$ :

(a) If $Y_{t}=0$, then pick a uniformly random vertex-copy from amongst all unmatched vertexcopies; let $u$ denote the vertex of which it is a copy. $C_{t+1}:=C_{t} \cup\{u\} ; Y_{t+1}:=\operatorname{deg}(u)$.

(b) Else choose an arbitrary unmatched vertex-copy of any vertex $v \in C_{t}$. Pick a uniformly random vertex-copy from amongst all other unmatched vertex-copies; let $u$ denote the vertex of which it is a copy. Match these two vertex-copies; thus exposing $u v$ as an edge of $C_{t+1}$.

i. If $u \notin C_{t}$ then $C_{t+1}:=C_{t} \cup\{u\} ; Y_{t+1}:=Y_{t}+\operatorname{deg}(u)-2$.

ii. Else $C_{t+1}:=C_{t} ; Y_{t+1}:=Y_{t}-2$.

Note that $C_{t}$ is a Markov process, and hence $Y_{t}$ depends only on $C_{t-1}$, and not on the way that $C_{t-1}$ is exposed in the branching process. For $t \geq 0$ let

- $\eta_{t+1}:=Y_{t+1}-Y_{t}$.

- $D_{t}:=Y_{t}+\sum_{u \notin C_{t}} d_{u}$, the total number of unmatched vertex-copies remaining at time $t$.

- $v_{t}:=\emptyset$ if $C_{t-1}$ and $C_{t}$ have the same vertex set, and if not, then $v_{t}$ is the unique vertex in $C_{t} \backslash C_{t-1}$.

- $Q_{t}:=\frac{\sum_{u \notin C_{t}} d_{u}^{2}}{D_{t}-1}-2$, and $R_{t}:=\frac{4\left(Y_{t}-1\right)+\sum_{u \notin C_{t}} d_{u}\left(d_{u}-2\right)^{2}}{D_{t}-1}$.

Remark: Since every vertex has degree at least one, $D_{t}>0$ for every $t$ until the procedure halts. Since the total number of vertex-copies is even, $D_{t}$ is even. Therefore $D_{t}>1$ and so $Q_{t}, R_{t}$ are well-defined.

Note that $Q_{t}$ and $R_{t}$ begin at $Q_{0} \approx Q$ and $R_{0} \approx R$. Furthermore, for $u \notin C_{t}, \operatorname{Pr}\left[v_{t+1}=u\right]=\frac{d_{u}}{D_{t}-1}$, and so if $Y_{t}>0$ then the expected change in $Y_{t}$ is

$$
\mathbb{E}\left[\eta_{t+1} \mid C_{t}\right]=\left(\sum_{u \notin C_{t}} \operatorname{Pr}\left[v_{t+1}=u\right] \times d_{u}\right)-2=\frac{\sum_{u \notin C_{t}} d_{u}^{2}}{D_{t}-1}-2=Q_{t} .
$$

If $Q_{t}$ remains approximately $Q$, then $Y_{t}$ is a random walk with drift approximately $Q$. So if $Q<0$ then we expect $Y_{t}$ to keep returning to zero quickly, and hence we only discover small components. But if $Q>0$ then we expect $Y_{t}$ to grow large; i.e. we expect to discover a large component. This is the intuition behind the main result of [17]. 
The parameter $R_{t}$ measures the expected value of the square of the change in $Y_{t}$, if $Y_{t}>0$ :

$$
\mathbb{E}\left[\eta_{t+1}^{2} \mid C_{t}\right]=\operatorname{Pr}\left[v_{t+1}=\emptyset\right] \times 4+\sum_{u \notin C_{t}} \operatorname{Pr}\left[v_{t+1}=u\right] \times\left(d_{u}-2\right)^{2}=\frac{4\left(Y_{t}-1\right)+\sum_{u \notin C_{t}} d_{u}\left(d_{u}-2\right)^{2}}{D_{t}-1}=R_{t} .
$$

If $Y_{t}=0$, then the expected values of $\eta_{t+1}$ and $\eta_{t+1}^{2}$ are not equal to $Q_{t}, R_{t}$, as in this case we have

$$
\mathbb{E}\left[\eta_{t+1} \mid C_{t}\right]=\frac{\sum_{u \notin C_{t}} d_{u}^{2}}{D_{t}}
$$

and, recalling from the above remark that $D_{t}>1$,

$$
\mathbb{E}\left[\eta_{t+1}^{2} \mid C_{t}\right]=\frac{\sum_{u \notin C_{t}} d_{u}^{3}}{D_{t}} \geq R_{t} \times \frac{D_{t}-1}{D_{t}} \geq \frac{R_{t}}{2} .
$$

Note that, for $Y_{t}>0$, the expected change in $Q_{t}$ is approximately:

$$
\mathbb{E}\left[Q_{t+1}-Q_{t} \mid C_{t}\right] \approx-\sum_{u \notin C_{t}} \operatorname{Pr}\left[v_{t+1}=u\right] \times \frac{d_{u}^{2}}{D_{t}-1}=-\frac{\sum_{u \notin C_{t}} d_{u}^{3}}{\left(D_{t}-1\right)^{2}}
$$

which, as long as $D_{t}=n-o(n)$, is asymptotically of the same order as $-\frac{R_{t}}{n}$. So if $R_{t}$ remains approximately $R$, then $Q_{t}$ will have a drift of roughly $-\frac{R}{n}$; i.e. the branching factor will decrease at approximately that rate. So amongst degree sequences with the same value of $Q$, we should expect those with large $R$ to have $\left|\mathcal{C}_{\max }\right|$ smaller. This explains why $\left|\mathcal{C}_{\max }\right|$ is a function of both $Q$ and $R$ in Theorem 1.1.

Finally, note that since $D_{t}$ decreases by at most 2 during any one step, we have

$$
D_{t} \geq 2|E|-2 t \text {. }
$$

\section{Concentration of $Q_{t}$ and $R_{t}$}

In this section, we estimate the expected values of $Q_{t}$ and $R_{t}$ and show that they are concentrated. We begin with $R_{t}$.

Lemma 3.1 For each $1 \leq t \leq \frac{\zeta}{400} \frac{n}{\Delta}$,

$$
\operatorname{Pr}\left[\left|R_{t}-R\right| \geq R / 2\right]<n^{-10} .
$$

Proof. It would be convenient if $R_{t}, R_{t-1}$ had the same denominator. So for $t \geq 1$ we define

$$
\tilde{R}_{t}:=\frac{4\left(Y_{t}-1\right)+\sum_{u \notin C_{t}} d_{u}\left(d_{u}-2\right)^{2}}{D_{t-1}-1}=R_{t} \frac{D_{t}-1}{D_{t-1}-1} .
$$

Note that $\left|D_{t}-D_{t-1}\right| \leq 2$ and $Y_{t} \leq \Delta t$, and that for $t \leq \frac{\zeta}{400} \frac{n}{\Delta}<\frac{1}{2}|E|$, we have $D_{t}, D_{t-1}>|E|$ by (5). Hence, applying Lemma 2.1, we obtain that for sufficiently large $n$,

$$
\begin{aligned}
\left|R_{t}-\tilde{R}_{t}\right| & =\left(4\left(Y_{t}-1\right)+\sum_{u \notin C_{t}} d_{u}\left(d_{u}-2\right)^{2}\right)\left|\frac{1}{D_{t}-1}-\frac{1}{D_{t-1}-1}\right| \\
& =\left(4\left(Y_{t}-1\right)+\sum_{u \notin C_{t}} d_{u}\left(d_{u}-2\right)^{2}\right) \frac{\left|D_{t}-D_{t-1}\right|}{\left(D_{t}-1\right)\left(D_{t-1}-1\right)} \\
& \leq 2 \frac{4\left(Y_{t}-1\right)+\sum_{u \notin C_{t}} d_{u}\left(d_{u}-2\right)^{2}}{|E|^{2}} \leq \frac{8 \Delta \times \frac{\zeta}{400} \frac{n}{\Delta}}{|E|^{2}}+\frac{4 R}{|E|}<\frac{5 R}{|E|} .
\end{aligned}
$$


Using again the fact that $D_{t-1}-1 \geq|E|$, we have for $n$ sufficiently large:

$$
\begin{aligned}
\left|\mathbb{E}\left[\tilde{R}_{t}-R_{t-1} \mid C_{t-1}\right]\right| & =\left|\mathbb{E}\left[\frac{4\left(Y_{t}-Y_{t-1}\right)}{D_{t-1}-1} \mid C_{t-1}\right]-\sum_{u \notin C_{t-1}} \operatorname{Pr}\left[v_{t}=u\right] \frac{d_{u}\left(d_{u}-2\right)^{2}}{D_{t-1}-1}\right| \\
& \leq \frac{4 \Delta}{|E|}+\sum_{u \notin C_{t-1}} \frac{d_{u}^{2}\left(d_{u}-2\right)^{2}}{\left(D_{t-1}-1\right)^{2}} \leq \frac{4 \Delta}{|E|}+\frac{2 \Delta R}{|E|}<\frac{20}{\zeta} \frac{\Delta R}{|E|},
\end{aligned}
$$

which together with (6) shows

$$
\left|\mathbb{E}\left[R_{t}-R_{t-1} \mid C_{t-1}\right]\right| \leq \frac{20}{\zeta} \frac{\Delta R}{|E|}+\frac{5 R}{|E|} \leq \frac{40}{\zeta} \frac{\Delta R}{|E|} .
$$

Using the fact that $(a+b)^{2} \leq 2 a^{2}+2 b^{2}$ we similarly obtain:

$$
\begin{aligned}
\mathbb{E}\left[\left|\tilde{R}_{t}-R_{t-1}\right|^{2} \mid C_{t-1}\right] & \leq 2 \mathbb{E}\left[\left(\frac{4\left(Y_{t}-Y_{t-1}\right)}{D_{t-1}-1}\right)^{2} \mid C_{t-1}\right]+2 \sum_{u \notin C_{t-1}} \operatorname{Pr}\left[v_{t}=u\right]\left(\frac{d_{u}\left(d_{u}-2\right)^{2}}{D_{t-1}-1}\right)^{2} \\
& \leq 2 \frac{16 \Delta^{2}}{|E|^{2}}+2 \sum_{u \notin C_{t-1}} \frac{d_{u}^{3}\left(d_{u}-2\right)^{4}}{\left(D_{t-1}-1\right)^{3}}<\frac{32 \Delta^{2}}{|E|^{2}}+\frac{4 \Delta^{4} R}{|E|^{2}}<\frac{150}{\zeta} \frac{\Delta^{4} R}{|E|^{2}}
\end{aligned}
$$

We conclude from this, (6) and Lemma 2.1(b) that

$$
\begin{aligned}
\operatorname{Var}\left[R_{t} \mid C_{t-1}\right] & \leq \mathbb{E}\left[\left|R_{t}-R_{t-1}\right|^{2} \mid C_{t-1}\right] \leq 2 \mathbb{E}\left[\left|\tilde{R}_{t}-R_{t-1}\right|^{2} \mid C_{t-1}\right]+2 \mathbb{E}\left[\left|R_{t}-\tilde{R}_{t}\right|^{2} \mid C_{t-1}\right] \\
& \leq 2 \frac{150}{\zeta} \frac{\Delta^{4} R}{|E|^{2}}+2(5)^{2} \frac{R^{2}}{|E|^{2}} \leq \frac{300}{\zeta} \frac{\Delta^{4} R}{|E|^{2}}+100 \frac{R \Delta}{|E|^{2}}<\frac{500}{\zeta} \frac{\Delta^{4} R}{|E|^{2}} .
\end{aligned}
$$

Note that by (7) and the bound $\Delta \leq n^{1 / 3} R^{1 / 3} / \ln n$, for $0 \leq t \leq \frac{\zeta}{400} \frac{n}{\Delta}$ and $n$ sufficiently large, we have:

$$
\left|R-\mathbb{E} R_{t}\right| \leq\left|R-R_{0}\right|+\left|R_{0}-\mathbb{E} R_{t}\right| \leq \frac{4 \Delta+\Delta(\Delta-2)^{2}}{2|E|}+t \times \frac{40}{\zeta} \frac{\Delta R}{|E|}<R / 4 .
$$

Applying (6), we have

$$
\left|R_{t}-R_{t-1}\right| \leq\left|R_{t}-\tilde{R}_{t}\right|+\left|\tilde{R}_{t}-R_{t-1}\right| \leq \frac{5 R}{|E|}+\frac{4 \Delta+\Delta(\Delta-2)^{2}}{D_{t-1}-1}<\frac{10 \Delta^{3}}{|E|} .
$$

Now by (8), (9), (10), and Theorem 2.3 and the bound $\Delta \leq n^{1 / 3} R^{1 / 3} / \ln n$ :

$$
\begin{aligned}
\operatorname{Pr}\left[\left|R_{t}-R\right| \geq \frac{R}{2}\right] & \leq \operatorname{Pr}\left[\left|R_{t}-\mathbb{E} R_{t}\right| \geq \frac{R}{4}\right] \leq e^{-\frac{(R / 4)^{2}}{2\left(\frac{R}{4} \frac{10 \Delta^{3}}{|E|}+\frac{500}{\zeta} \frac{\Delta^{4} R}{|E|^{2}}\right)}}<e^{-\frac{R|E|}{200 \Delta^{3}}} \\
& <e^{-(\ln n)^{3}}<n^{-10}
\end{aligned}
$$

for sufficiently large $n$.

Next we turn to $Q_{t}$ :

Lemma 3.2 For each $1 \leq t \leq \frac{\zeta}{1000} \frac{|Q| n}{R}+2 n^{2 / 3} R^{-1 / 3}$,

$$
\operatorname{Pr}\left[\left|Q_{t}-Q\right|>\frac{1}{2}|Q|+\frac{800}{\zeta} n^{-1 / 3} R^{2 / 3}\right] \leq n^{-10} .
$$


Proof. Again, we make the denominators the same by setting

$$
\tilde{Q}_{t}:=\frac{\sum_{u \notin C_{t}} d_{u}^{2}}{D_{t-1}-1}-2
$$

Using the same argument as for (6), and applying Lemma 2.1(a), we obtain that for sufficiently large $n$,

$$
\left|Q_{t}-\tilde{Q}_{t}\right| \leq 2 \frac{\sum_{u \notin C_{t}} d_{u}^{2}}{|E|^{2}} \leq \frac{4|Q|+8}{|E|}<\frac{10}{|E|}
$$

Trivially

$$
\left|Q_{t}-Q_{t-1}\right| \leq\left|Q_{t}-\tilde{Q}_{t}\right|+\left|\tilde{Q}_{t}-Q_{t-1}\right| \leq \frac{\Delta^{2}}{|E|}+\frac{10}{|E|}<\frac{20 \Delta^{2}}{|E|} .
$$

In what follows, we use the facts that $2|E| \geq D_{t-1}-1 \geq 2|E|-2 t+1>|E|$ and $D_{t-1} \geq Y_{t-1}$. Note that whether $Y_{t-1}>0$ or $Y_{t-1}=0$, for sufficiently large $n$, by Lemma 2.1(a) we always have

$$
\begin{aligned}
\mathbb{E}\left[\tilde{Q}_{t}-Q_{t-1} \mid C_{t-1}\right] & =-\sum_{u \notin C_{t-1}} \operatorname{Pr}\left[v_{t}=u\right] \frac{d_{u}^{2}}{D_{t-1}-1}=-\sum_{u \notin C_{t-1}} \frac{d_{u}^{3}}{\left(D_{t-1}-1\right)^{2}} \\
& <-\frac{4\left(Y_{t-1}-1\right)+\sum_{u \notin C_{t-1}} d_{u}\left(d_{u}-2\right)^{2}-4\left(Y_{t-1}-1\right)}{\left(D_{t-1}-1\right)^{2}} \\
& \leq-\frac{R_{t-1}-4}{D_{t-1}-1} \leq-\frac{R_{t-1}}{2|E|}+\frac{4}{|E|} .
\end{aligned}
$$

Combining this with (11) we have

$$
\mathbb{E}\left[Q_{t}-Q_{t-1} \mid C_{t-1}\right] \leq-\frac{R_{t-1}}{2|E|}+\frac{4}{|E|}+\frac{10}{|E|}<-\frac{R_{t-1}-30}{2|E|} .
$$

For $n$, and hence $|E|$, sufficiently large:

$$
Q_{0}-Q=\frac{d_{v}^{2}}{2|E|-1}+\sum_{u \in G} d_{u}^{2}\left(\frac{1}{2|E|-1}-\frac{1}{2|E|}\right)=\frac{d_{v}^{2}}{2|E|-1}+\frac{(Q+2)}{2|E|-1}<\frac{\Delta^{2}}{|E|}
$$

Now (9), (13), Lemma $2.1(\mathrm{a}, \mathrm{b})$ and the bound $\Delta \leq n^{1 / 3} R^{1 / 3} / \ln n$ imply that for $t \leq \frac{\zeta}{1000} \frac{|Q| n}{R}+$ $2 n^{2 / 3} R^{-1 / 3}$,

$$
\begin{aligned}
\mathbb{E}\left[Q_{t}-Q\right] & \leq\left|Q-Q_{0}\right|+\mathbb{E}\left[Q_{t}-Q_{0}\right] \leq \frac{\Delta^{2}}{|E|}+\left(\frac{30 t}{2|E|}-\sum_{j=0}^{t-1} \mathbb{E} \frac{R_{j}}{2|E|}\right) \leq \frac{\Delta^{2}}{|E|}+\frac{30 t}{2|E|} \\
& \leq 2 n^{-1 / 3} R^{2 / 3}(\ln n)^{-2}+\frac{|Q|}{4}+60 n^{-1 / 3} R^{-1 / 3} \leq \frac{|Q|}{4}+\frac{400}{\zeta} n^{-1 / 3} R^{2 / 3}
\end{aligned}
$$

Furthermore

$$
\begin{aligned}
\mathbb{E}\left[\tilde{Q}_{t}-Q_{t-1} \mid C_{t-1}\right] & =-\sum_{u \notin C_{t-1}} \frac{d_{u}^{3}}{\left(D_{t-1}-1\right)^{2}} \\
& \geq-\frac{9 \sum_{u \notin C_{t-1}} d_{u}\left(d_{u}-2\right)^{2}+\sum_{u: d_{u}=2} 8}{|E|^{2}} \\
& >-\frac{18 R+8}{|E|} \geq-\frac{50}{\zeta} \frac{R}{|E|}
\end{aligned}
$$


which together with (11) shows that

$$
\mathbb{E}\left[Q_{t}-Q_{t-1} \mid C_{t-1}\right] \geq-\frac{50}{\zeta} \frac{R}{|E|}-\frac{10}{|E|} \geq-\frac{90}{\zeta} \frac{R}{|E|},
$$

and hence for $1 \leq t \leq \frac{\zeta}{1000} \frac{|Q| n}{R}+2 n^{2 / 3} R^{-1 / 3}$, using the bound $\Delta \leq n^{1 / 3} R^{1 / 3} / \ln n$,

$$
\mathbb{E}\left[Q_{t}-Q\right] \geq \mathbb{E}\left[Q_{t}-Q_{0}\right]-\left|Q-Q_{0}\right| \geq-\frac{90}{\zeta} \frac{t R}{|E|}-\Delta^{2} n^{-1}>-\frac{|Q|}{4}-\frac{400}{\zeta} n^{-1 / 3} R^{2 / 3} .
$$

Using similar arguments we obtain

$$
\begin{aligned}
\mathbb{E}\left[\left|\tilde{Q}_{t}-Q_{t-1}\right|^{2} \mid C_{t-1}\right] & =\sum_{u \notin C_{t-1}} \operatorname{Pr}\left[v_{t}=u\right] \frac{d_{u}^{4}}{\left(D_{t-1}-1\right)^{2}}=\sum_{u \notin C_{t-1}} \frac{d_{u}^{5}}{\left(D_{t-1}-1\right)^{3}} \\
& \leq \Delta^{2} \sum_{u \notin C_{t-1}} \frac{d_{u}^{3}}{|E|^{3}} \leq \frac{\Delta^{2}}{|E|^{2}} \frac{9 \sum_{u \notin C_{t-1}} d_{u}\left(d_{u}-2\right)^{2}+\sum_{u: d_{u}=2} 8}{|E|} \\
& \leq \frac{50}{\zeta} \frac{\Delta^{2} R}{|E|^{2}} \leq \frac{200}{\zeta} \frac{\Delta^{2} R}{n^{2}}
\end{aligned}
$$

As in (8), this, (11) and Lemma 2.1(b) yield

$$
\begin{aligned}
\operatorname{Var}\left[Q_{t} \mid C_{t-1}\right] & \leq \mathbb{E}\left[\left|Q_{t}-Q_{t-1}\right|^{2} \mid C_{t-1}\right] \leq 2 \mathbb{E}\left[\left|\tilde{Q}_{t}-Q_{t-1}\right|^{2} \mid C_{t-1}\right]+2 \mathbb{E}\left[\left|Q_{t}-\tilde{Q}_{t}\right|^{2} \mid C_{t-1}\right] \\
& \leq 2 \frac{200}{\zeta} \frac{\Delta^{2} R}{n^{2}}+2 \frac{100}{|E|^{2}}<\frac{2000}{\zeta} \frac{\Delta^{2} R}{n^{2}} .
\end{aligned}
$$

By (14), (15) and (12), we can apply Theorem 2.3 with $\rho=\frac{1}{4}|Q|+\frac{400}{\zeta} n^{-1 / 3} R^{2 / 3}$ and $M=\frac{20 \Delta^{2}}{|E|}<$ $40 n^{-1 / 3} R^{2 / 3}(\ln n)^{-2}$. Similarly, (16) allows us to take $\sigma_{i}^{2}=\frac{2000}{\zeta} \frac{\Delta^{2} R}{n^{2}}<\frac{2000}{\zeta} n^{-4 / 3} R^{5 / 3}(\ln n)^{-2}$. This yields:

$$
\begin{aligned}
\operatorname{Pr}\left[\left|Q_{t}-Q\right| \geq \frac{1}{2}|Q|+\frac{800}{\zeta} n^{-1 / 3} R^{2 / 3}\right] & \leq 2 e^{-\frac{\left(\frac{1}{4}|Q|+\frac{400}{\zeta} n^{-1 / 3} R^{2 / 3}\right)^{2}}{2\left(40 n^{-1 / 3} R^{2 / 3} \times\left(\frac{1}{4}|Q|+\frac{400}{\zeta} n^{-1 / 3} R^{2 / 3}\right)+t \times \frac{2000}{\zeta} n^{-4 / 3} R^{5 / 3}\right)(\ln n)^{-2}}} \\
& \leq 2 e^{-\frac{\left(\frac{1}{4}|Q|+\frac{400}{\zeta} n^{-1 / 3} R^{2 / 3}\right)^{2}(\ln n)^{2}}{80 n^{-1 / 3} R^{2 / 3} \times\left(\frac{1}{4}|Q|+\frac{400}{\zeta} n^{-1 / 3} R^{2 / 3}\right)+4|Q| n^{-1 / 3} R^{2 / 3}+\frac{8000}{\zeta} n^{-2 / 3} R^{4 / 3}}} .
\end{aligned}
$$

To bound this, note that:

$$
\begin{gathered}
\frac{\left(\frac{1}{4}|Q|+\frac{400}{\zeta} n^{-1 / 3} R^{2 / 3}\right)^{2}}{80 n^{-1 / 3} R^{2 / 3} \times\left(\frac{1}{4}|Q|+\frac{400}{\zeta} n^{-1 / 3} R^{2 / 3}\right)}=\frac{\frac{1}{4}|Q|+\frac{400}{\zeta} n^{-1 / 3} R^{2 / 3}}{80 n^{-1 / 3} R^{2 / 3}} \geq \frac{5}{\zeta} . \\
\frac{\left(\frac{1}{4}|Q|+\frac{400}{\zeta} n^{-1 / 3} R^{2 / 3}\right)^{2}}{4|Q| n^{-1 / 3} R^{2 / 3}} \geq \frac{\frac{400}{\zeta} n^{-1 / 3} R^{2 / 3}}{16 n^{-1 / 3} R^{2 / 3}}=\frac{25}{\zeta} . \\
\frac{\left(\frac{1}{4}|Q|+\frac{400}{\zeta} n^{-1 / 3} R^{2 / 3}\right)^{2}}{\frac{8000}{\zeta} n^{-2 / 3} R^{1 / 3}} \geq \frac{\left(n^{-1 / 3} R^{2 / 3}\right)^{2}}{\zeta n^{-2 / 3} R^{4 / 3}}=\frac{1}{\zeta} .
\end{gathered}
$$

These yield:

$$
\operatorname{Pr}\left[\left|Q_{t}-Q\right| \geq \frac{1}{2}|Q|+\frac{800}{\zeta} n^{-1 / 3} R^{2 / 3}\right] \leq 2 e^{-\frac{(\ln n)^{2}}{3 \zeta}}<n^{-10},
$$

for $n$ sufficiently large. 


\section{Proof of Theorem 1.2}

We start by analyzing the subcritical phase; i.e. when $Q<-\omega(n) n^{-1 / 3} R^{2 / 3}$, where $\omega(n)$ grows with $n$.

First we show that with high probability, there are no components of size greater than $O(\sqrt{n /|Q|})$. The proof will be a simple application of the Optional Stopping Theorem.

Proof of Theorem 1.2(a). Fix any $\epsilon>0$ and set $B=\frac{4}{\sqrt{\epsilon}}$ and $T=B \sqrt{n /|Q|}$. For a given vertex $v$, we will bound the probability that $v$ lies in a component of size at least $T$ by analyzing the branching process beginning at vertex $v$ and bounding the probability that $Y_{t}$ does not return to zero before time $T$.

Note that for $n$ sufficiently large, $T<n^{2 / 3} R^{-1 / 3}$. So Lemma 3.2, implies that, with high probability,

$$
Q_{t} \leq \frac{1}{2} Q+\frac{800}{\zeta} n^{1 / 3} R^{2 / 3}<\frac{1}{4} Q,
$$

for every $t \leq T$. We define the stopping time

$$
\gamma:=\min \left\{t:\left(Y_{t}=0\right),\left(Q_{t}>\frac{1}{4} Q\right) \text { or }(t=T)\right\} .
$$

Lemma 3.2 will show us that, with high probability, we will not have $Q_{\gamma}>\frac{1}{4} Q$. So by upper bounding $\operatorname{Pr}(\gamma=T)$, we can obtain a good lower bound on $\operatorname{Pr}\left(Y_{t}=0\right)$ which, in turn, is a lower bound on the probability that $Y_{t}$ reaches zero before time $T$.

For $t \leq \gamma$, we have $Q_{t-1} \leq \frac{1}{4} Q$. We also have $Y_{t-1}>0$ and so $\mathbb{E}\left(\eta_{t}\right)$ is as in (1). Therefore we have:

$$
\mathbb{E}\left(Y_{t}-Y_{t-1}\right)=Q_{t-1} \leq \frac{1}{4} Q,
$$

and so $Y_{\min (t, \gamma)}-\frac{1}{4} Q \min (t, \gamma)$ is a supermartingale. Applying the Optional Stopping Theorem to $Y_{\min (t, \gamma)}-\frac{1}{4} Q \min (t, \gamma)$ with stopping times $\tau:=\gamma$ and bound $T:=T$ yields that

$$
\mathbb{E}\left(Y_{\gamma}-\frac{1}{4} Q \gamma\right) \leq Y_{0}=d_{v} .
$$

Since $Q<0$, this implies:

$$
\mathbb{E}(\gamma) \leq \frac{4\left(d_{v}-\mathbb{E}\left(Y_{\gamma}\right)\right)}{|Q|} \leq \frac{4 d_{v}}{|Q|}
$$

and so $\operatorname{Pr}(\gamma=T) \leq \frac{4 d_{v}}{|Q| T}$. By Lemma 3.2, $\operatorname{Pr}\left(Q_{\gamma}>\frac{1}{4} Q\right)<n^{-10}$ and so:

$$
\operatorname{Pr}\left(Y_{\gamma} \neq 0\right) \leq \frac{4 d_{v}}{|Q| T}+n^{-10}<\frac{5 d_{v}}{|Q| T},
$$

for $n$ sufficiently large.

Let $Z$ be the number of vertices lying in components of size at least $T$. Recalling that $\sum_{v} d_{v}=$ $2|E|<3 n$ by Lemma 2.1(a), we have

$$
\begin{aligned}
\operatorname{Pr}\left[\left|\mathcal{C}_{\max }\right| \geq T\right] & \leq \operatorname{Pr}[Z \geq T] \leq \frac{\mathbb{E}[Z]}{T} \leq \frac{1}{T} \sum_{v \in V} \operatorname{Pr}\left[\mathcal{C}_{v} \geq T\right] \\
& \leq \frac{1}{T} \sum_{v \in V} \frac{5 d_{v}}{|Q| T}<\frac{16 n}{|Q| T^{2}}=\frac{16}{B^{2}}=\epsilon
\end{aligned}
$$

This proves that Theorem 1.2(a) holds for a random configuration. Proposition 2.2 implies that it holds for a random graph.

Next we show that the random graph will, with probability at least $\epsilon$, have no components with at least two cycles. The following helpful fact bounds the probability that specific pairs of vertex-copies are joined in our random configuration: 
Proposition 4.1 Specify any $\ell$ pairs of vertex-copies. The probability that those pairs are joined is at most $\frac{(|E|-1-\ell) !}{2^{\ell}(|E|-1) !}$.

Proof. The number of ways of pairing $2 r$ points is $\frac{(2 r) !}{2^{r} r !}$. So the ratio of the number of configurations with those $\ell$ pairs joined to the total number of configurations is

$$
\begin{aligned}
\frac{(2|E|-2 \ell) ! / 2^{|E|-\ell}(|E|-\ell) !}{(2|E|) ! / 2^{|E|}|E| !} & =\frac{2^{\ell}|E|(|E|-1) \ldots(|E|-\ell+1)}{(2|E|) \ldots(2|E|-2 \ell+1)} \\
& =\frac{1}{(2|E|-1)(2|E|-3) \ldots(2|E|-2 \ell+1)} \\
& <\frac{1}{2^{\ell}(|E|-1)(|E|-2) \ldots(|E|-\ell)} .
\end{aligned}
$$

We will also use:

Proposition 4.2 For any $w_{1}, \ldots, w_{n} \geq 0$, the average over all subsets $\left\{x_{1}, \ldots, x_{\ell}\right\} \subset\{1, \ldots, n\}$ of $\prod_{i=1}^{\ell} w_{x_{i}}$ is at most the average over all $\ell$-tuples $\left(x_{1}, \ldots, x_{\ell}\right) \in\{1, \ldots, n\}^{\ell}$ of $\prod_{i=1}^{\ell} w_{x_{i}}$.

Proof. It is trivially true if $n=1$. Note that the average over all $\ell$-tuples $\left(x_{1}, \ldots, x_{\ell}\right) \in\{1, \ldots, n\}^{\ell}$ of $\prod_{i=1}^{\ell} w_{x_{i}}$ is equal to $n^{-\ell}\left(\sum_{i=1}^{n} w_{i}\right)^{\ell}$ and hence is determined by $\sum_{i=1}^{n} w_{i}$. The proposition now follows from the easy observations: (i) the two averages are equal if $w_{1}=\ldots=w_{n}$ and (ii) if $w_{i} \leq w_{j}$ then replacing $w_{i}, w_{j}$ by $w_{i}-\epsilon, w_{j}+\epsilon$ decreases $w_{i} w_{j}$ and hence decreases the average over all subsets $\left\{x_{1}, \ldots, x_{\ell}\right\} \subset\{1, \ldots, n\}$ of $\prod_{i=1}^{\ell} w_{x_{i}}$.

Proof of Theorem 1.2(b) As noted by Karonski for the proof of the very similar Lemma 1(iii) of [16]: if a component contains at least two cycles then it must contain at least one of the following two subgraphs:

- $W_{1}$ - two vertices $u, v$ that are joined by three paths, where the paths are vertex-disjoint except for at their endpoints.

- $W_{2}$ - two edge-disjoint cycles, one containing $u$ and the other containing $v$, and a $(u, v)$-path that is edge-disjoint from the cycles. We allow $u=v$ in which case the path has length zero.

In particular, if it contains two cycles that share more than one vertex, then it is easy to see that it must contain a pair of cycles that form $W_{1}$. And if it contains two cycles that share at most one vertex, then those cycles plus a shortest path between them must form $W_{2}$.

We will prove that the expected number of such subgraphs of size at most $\frac{1}{4} n$ is less than $\frac{1}{2} \epsilon$. By part (a) (after rescaling $\epsilon$ ), the probability that there is any component of size greater than $\frac{1}{4} n$ is less than $\frac{1}{2} \epsilon$. This proves the theorem. We begin with the expected number of $W_{1}$ 's.

Specify $u, v$, the number of internal vertices on each path $-\ell_{1}, \ell_{2}, \ell_{3}$, and the internal vertices $x_{1}, \ldots, x_{\ell_{1}+\ell_{2}+\ell_{3}}$, in order along the paths. Next specify exactly which vertex-copies are paired to form the edges of $W_{1}$; the number of choices is $d_{u}\left(d_{u}-1\right)\left(d_{u}-2\right) \times d_{v}\left(d_{v}-1\right)\left(d_{v}-2\right) \times \prod_{i=1}^{\ell_{1}+\ell_{2}+\ell_{3}} d_{x_{i}}\left(d_{x_{i}}-1\right)$. Therefore, applying Proposition 4.1, the expected number of such subgraphs is at most:

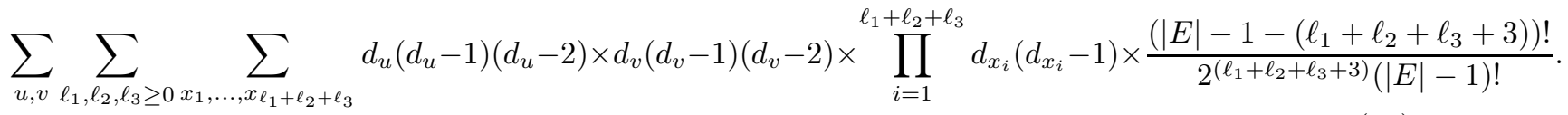


Setting $\ell=\ell_{1}+\ell_{2}+\ell_{3}$ and $w_{x_{i}}=d_{x_{i}}\left(d_{x_{i}}-1\right)$, Proposition 4.2 implies that:

$$
\sum_{x_{1}, \ldots, x_{\ell}} \prod_{i=1}^{\ell} w_{x_{i}} \times \frac{(n-\ell) !}{n !} \leq\left(\frac{\sum_{x \in V(G)} w_{x}}{n}\right)^{\ell} .
$$

By Lemma 2.1(a), we have $|E|-1<n($ since $Q<0)$ and so $\frac{(|E|-1-\ell) !}{(|E|-1) !} \times(|E|-1)^{\ell}<\frac{(n-\ell) !}{n !} \times n^{\ell}$. This implies:

$$
\sum_{x_{1}, \ldots, x_{\ell}} \prod_{i=1}^{\ell} w_{x_{i}} \times \frac{(|E|-1-\ell) !}{(|E|-1) !}<\left(\frac{\sum_{x \in V(G)} w_{x}}{|E|-1}\right)^{\ell}=\left(\frac{\sum_{x \in V(G)} w_{x}}{|E|}\right)^{\ell} \times\left(1+\frac{1}{|E|-1}\right)^{\ell} .
$$

Since $\ell \leq \frac{1}{4} n$ and $|E| \geq \frac{1}{2} n$ (by Lemma 2.1(a)), we have $(|E|-1-(\ell+1))(|E|-1-(\ell+2))(|E|-$ $1-(\ell+3))>\frac{1}{10}|E|^{3}$ and $\left(1+\frac{1}{|E|-1}\right)^{\ell}<e^{n /(4|E|-4)}<e$. Thus by (17), the expected number of $W_{1}$ subgraphs is at most:

$$
\begin{aligned}
& \frac{10 e}{8|E|^{3}}\left(\sum_{u \in V(G)} d_{u}\left(d_{u}-1\right)\left(d_{u}-2\right)\right)^{2}\left(\frac{\sum_{x \in V(G)} d_{x}\left(d_{x}-1\right)}{2|E|}\right)^{\ell_{1}+\ell_{2}+\ell_{3}} \\
< & \frac{5 e R^{2}}{|E|} \sum_{\ell_{1}, \ell_{2}, \ell_{3} \geq 0}(1+Q)^{\ell_{1}+\ell_{2}+\ell_{3}} \\
= & \frac{5 e R^{2}}{|E|}\left(\sum_{\ell \geq 0}(1+Q)^{\ell}\right)^{3} \\
= & \frac{5 e R^{2}}{|E||Q|^{3}}<\frac{e}{20 \omega(n)^{3}}<\frac{1}{4} \epsilon .
\end{aligned}
$$

A nearly identical argument shows that the expected number of subgraphs of type $W_{2}$ with $u \neq v$ is also at most $\frac{1}{4} \epsilon$-this time $\ell_{1}, \ell_{2}, \ell_{3}$ denote the number of vertices, other than $u, v$ on the two cycles and the path. In the case where $u=v,\left(\sum_{u} d_{u}\left(d_{u}-1\right)\left(d_{u}-2\right)\right)^{2}$ is replaced with $\sum_{u} d_{u}\left(d_{u}-1\right)\left(d_{u}-2\right)\left(d_{u}-3\right)$, which is smaller.

This proves that Theorem 1.2(b) holds for a random configuration. Proposition 2.2 implies that it holds for a random graph.

Remark: Note that in the proof of part (b), Condition D was only used to (i) allow us to apply part (a) to show that the size of the largest component, and hence $\ell$, is at most $\frac{1}{4} n$, and (ii) switch from random configurations to random graphs. Step (i) could have been carried out without Condition D: any bound of the form $|E|-\Theta(|E|)$ would have sufficed, and we can obtain such a bound easily, eg. by arguing that with high probability, there are $\theta(|E|)$ components of size 2. Step (ii) can be carried out under much weaker conditions than Condition D.

\section{Proof of Theorem 1.1(b)}

In this section we turn to the critical range of $Q$; i.e. $-\lambda n^{-1 / 3} R^{2 / 3} \leq Q \leq \lambda n^{-1 / 3} R^{2 / 3}$. We will bound the probability that the size of the largest component is too big. Without loss of generality, we can assume that $\lambda>\frac{1600}{\zeta}$.

Our proof follows along the same lines as that of Theorem 1 (see also Theorem 7) of [19].

We wish to show that there exists a constant $B>1$ such that with probability at least $1-\epsilon$, the largest component has size at most $B n^{2 / 3} R^{-1 / 3}$. To do so, we set $T:=n^{2 / 3} R^{-1 / 3}$ and bound the 
probability that our branching process starting at a given vertex $v$ does not return to zero within $T$ steps.

Lemma 3.2 yields that, with high probability, $\left|Q_{t}-Q\right| \leq \frac{1}{2}|Q|+\frac{800}{\zeta} n^{-1 / 3} R^{2 / 3}$ for every $t \leq T$. Since we assume $\lambda>\frac{1600}{\zeta}$, this implies $Q_{t} \leq 2 \lambda n^{-1 / 3} R^{2 / 3}$.

The fact that the drift, $Q_{t}$, may be positive makes this case a bit trickier than that in the previous section, and so we need a more involved argument. It will be convenient to assume that $Y_{t}$ is bounded by $H:=\frac{1}{12 \lambda} n^{1 / 3} R^{1 / 3}$, so we add $Y_{t} \geq H$ to our stopping time conditions. We also need to add a condition corresponding to the concentration of $R$. Specifically, we define

$$
\gamma:=\min \left\{t:\left(Y_{t}=0\right),\left(Y_{t} \geq H\right),\left(Q_{t}>2 \lambda n^{-1 / 3} R^{2 / 3}\right),\left(\left|R_{t}-R\right|>R / 2\right) \text { or }(t=T)\right\} .
$$

Since $\Delta \leq n^{1 / 3} R^{1 / 3} / \ln n$, we have $T<\frac{\zeta}{400} \frac{n}{\Delta}$ for $n$ sufficiently large. So Lemmas 3.1 and 3.2 imply that, with high probability, we will not have $Q_{\gamma}>2 \lambda n^{-1 / 3} R^{2 / 3}$ or $\left|R_{\gamma}-R\right|>R / 2$. So by upper bounding $\operatorname{Pr}\left(Y_{\gamma} \geq H\right)$ and $\operatorname{Pr}(\gamma=T)$, we can obtain a good lower bound on $\operatorname{Pr}\left(Y_{t}=0\right)$ which, in turn, is a lower bound on $Y_{t}$ reaching zero before reaching $H$.

For $t \leq \gamma$, we have $Q_{t-1} \leq 2 \lambda n^{-1 / 3} R^{2 / 3}$ and so:

$$
H Q_{t-1} \leq \frac{1}{6} R
$$

For $t \leq \gamma$, we also have $Y_{t-1}>0$ and so $\mathbb{E}\left(\eta_{t}\right)$ and $\mathbb{E}\left(\eta_{t}^{2}\right)$ are as in (1) and (2). We also have $R_{t-1} \geq \frac{1}{2} R$ and (19). For small enough $x \geq 0, e^{-x} \geq 1-x+x^{2} / 3$. So for $n$ sufficiently large, $\left|\eta_{t} / H\right| \leq(2+\Delta) / H<(\ln n)^{-1}$ is small enough to yield:

$$
\begin{aligned}
\mathbb{E}\left[e^{-\eta_{t} / H} \mid C_{t-1}\right] & \geq 1-\mathbb{E}\left[\frac{\eta_{t}}{H} \mid C_{t-1}\right]+\frac{1}{3} \mathbb{E}\left[\frac{\eta_{t}^{2}}{H^{2}} \mid C_{t-1}\right]=1-\frac{Q_{t-1}}{H}+\frac{R_{t-1}}{3 H^{2}} \\
& \geq 1-\frac{R}{6 H^{2}}+\frac{R}{6 H^{2}}=1 .
\end{aligned}
$$

This shows that $e^{-Y_{\min (t, \gamma)} / H}$ is a submartingale, and so we can apply the Optional Stopping Theorem with stopping time $\tau:=\gamma$. As $Y_{\gamma-1} \leq H$, we have $Y_{\gamma} \leq H+\Delta<2 H$. Recalling that we begin our branching process at vertex $v$ and applying $x / 4 \leq 1-e^{-x}$, for $0 \leq x \leq 2$, we have:

$$
e^{-d_{v} / H}=e^{-Y_{0} / H} \leq \mathbb{E} e^{-Y_{\gamma} / H} \leq \mathbb{E}\left[1-\frac{Y_{\gamma}}{4 H}\right],
$$

which, using the fact that for $x>0,1-e^{-x} \leq x$, implies

$$
\mathbb{E}\left[Y_{\gamma}\right] \leq 4 H\left(1-e^{-d_{v} / H}\right) \leq 4 d_{v} .
$$

In particular

$$
\operatorname{Pr}\left[Y_{\gamma} \geq H\right] \leq \frac{4 d_{v}}{H}
$$

Now we turn our attention to $\operatorname{Pr}(\gamma=T)$. We begin by bounding:

$$
\mathbb{E}\left[Y_{t}^{2}-Y_{t-1}^{2} \mid C_{t-1}\right]=\mathbb{E}\left[\left(\eta_{t}+Y_{t-1}\right)^{2}-Y_{t-1}^{2} \mid C_{t-1}\right]=\mathbb{E}\left[\eta_{t}^{2} \mid C_{t-1}\right]-2 \mathbb{E}\left[\eta_{t} Y_{t-1} \mid C_{t-1}\right] .
$$

For $t \leq \gamma$, we have $Y_{t-1}>0$ and so $\mathbb{E}\left[\eta_{t} \mid C_{t-1}\right]=Q_{t-1}$. Thus $\mathbb{E}\left[\eta_{t} Y_{t-1} \mid C_{t-1}\right]=Q_{t-1} Y_{t-1}$. Also, for $t \leq \gamma$, we must have $Y_{t-1}<H, R_{t-1} \geq \frac{1}{2} R$ and (19) so:

$$
\mathbb{E}\left[Y_{t}^{2}-Y_{t-1}^{2} \mid C_{t-1}\right] \geq R_{t-1}-2 H \max \left(Q_{t-1}, 0\right) \geq \frac{R}{2}-\frac{R}{3}=\frac{R}{6} .
$$

Thus $Y_{\min (t, \gamma)}^{2}-\frac{1}{6} R \min (t, \gamma)$ is a submartingale, and so by the Optional Stopping Theorem we have:

$$
\mathbb{E}\left[Y_{\gamma}^{2}-\frac{R \gamma}{6}\right] \geq Y_{0}^{2}=d_{v}^{2} \geq 0
$$


This, together with (20) and the fact (derived above) that $Y_{\gamma} \leq 2 H$, implies that

$$
\mathbb{E} \gamma \leq \frac{6}{R} \mathbb{E} Y_{\gamma}^{2} \leq \frac{12 H}{R} \mathbb{E} Y_{\gamma} \leq \frac{48 H d_{v}}{R},
$$

showing

$$
\operatorname{Pr}[\gamma=T] \leq \frac{48 H d_{v}}{R T}
$$

We conclude from (21), (22), and Lemmas 3.1 and 3.2 that, for $n$ sufficiently large,

$$
\begin{aligned}
\operatorname{Pr}\left[\left|\mathcal{C}_{v}\right| \geq T\right] & \leq \operatorname{Pr}\left[Y_{\gamma}>H\right]+\operatorname{Pr}[\gamma=T]+\operatorname{Pr}\left[Q_{t}>2 \lambda n^{-1 / 3} R^{2 / 3}\right]+\operatorname{Pr}\left[\left|R_{\gamma}-R\right|>R / 2\right] \\
& \leq \frac{4 d_{v}}{H}+\frac{48 H d_{v}}{R T}+T n^{-10}+T n^{-10} \\
& \leq 48 \lambda n^{-1 / 3} R^{-1 / 3} d_{v}+\frac{48 n^{1 / 3} R^{1 / 3} d_{v}}{12 \lambda n^{2 / 3} R^{2 / 3}}+2 T n^{-10}<50 \lambda n^{-1 / 3} R^{-1 / 3} d_{v}
\end{aligned}
$$

For some constant $B \geq 1$, let $N$ be the number of vertices lying in components of size at least $K:=$ $B n^{2 / 3} R^{-1 / 3} \geq T$. Recalling that $\sum_{v} d_{v}=2|E|<3 n$ by Lemma 2.1 (a), we have

$$
\begin{aligned}
\operatorname{Pr}\left[\left|\mathcal{C}_{\max }\right| \geq K\right] & \leq \operatorname{Pr}[N \geq K] \leq \frac{\mathbb{E}[N]}{K} \leq \frac{1}{K} \sum_{v \in V} \operatorname{Pr}\left[\mathcal{C}_{v} \geq K\right] \leq \frac{1}{K} \sum_{v \in V} \operatorname{Pr}\left[\mathcal{C}_{v} \geq T\right] \\
& \leq \frac{1}{K} \sum_{v \in V} 50 \lambda n^{-1 / 3} R^{-1 / 3} d_{v}=\frac{50 \lambda}{n B} \sum_{v} d_{v}<\frac{150 \lambda}{B}
\end{aligned}
$$

which can be made to be less than $\epsilon$ by taking $B$ to be sufficiently large. This proves that Theorem 1.1(b) holds for a random configuration. Proposition 2.2 implies that it holds for a random graph.

\section{Proof of Theorem 1.1(a)}

In this section we bound the probability that the size of the largest component is too small when $Q$ is in the critical range. Our proof follows along the same lines as that of Theorem 2 of [19].

Recall that we have $-\lambda n^{2 / 3} R^{2 / 3} \leq Q \leq \lambda n^{-1 / 3} R^{2 / 3}$. Without loss of generality, we can assume that $\lambda>\frac{1600}{\zeta}$.

We wish to show that there exists a constant $A>0$ such that with probability at least $1-\epsilon$, the largest component has size at least $A n^{2 / 3} R^{-1 / 3}$.

We will first show that, with sufficiently high probability, our branching process reaches a certain value $h$. Then we will show that, with sufficiently high probability, it will take at least $A n^{2 / 3} R^{-1 / 3}$ steps for it to get from $h$ to zero, and thus there must be a component of that size.

We set $T_{1}:=n^{2 / 3} R^{-1 / 3}$ and $T_{2}:=A n^{2 / 3} R^{-1 / 3}$. For $t \leq T_{1}+T_{2} \leq 2 n^{2 / 3} R^{-1 / 3}$ (for $A \leq 1$ ), Lemma 3.2 yields that, with high probability, $\left|Q_{t}-Q\right| \leq \frac{1}{2}|Q|+\frac{800}{\zeta} n^{-1 / 3} R^{2 / 3}$ and thus (since $\lambda>\frac{1600}{\zeta}$ )

$$
Q_{t} \geq-2 \lambda n^{-1 / 3} R^{2 / 3}
$$

We set

$$
h:=A^{1 / 4} n^{1 / 3} R^{1 / 3}
$$

so that if $Q_{t} \geq-2 \lambda n^{-1 / 3} R^{2 / 3}$ and $A<(16 \lambda)^{-4}$ then

$$
h Q_{t} \geq-2 \lambda A^{1 / 4} R \geq-\frac{R}{8} .
$$


We start by showing that $Y_{t}$ reaches $h$, with sufficiently high probability. To do so, we define $\tau_{1}$ analogously to $\gamma$ from Section 5, the only difference being that we allow $Y_{t}$ to return to zero before $t=\tau_{1}$.

$$
\tau_{1}=\min \left\{t:\left(Y_{t} \geq h\right),\left(Q_{t}<-2 \lambda n^{-1 / 3} R^{2 / 3}\right),\left(\left|R_{t}-R\right|>R / 2\right), \text { or }\left(t=T_{1}\right)\right\} .
$$

We wish to show that, with sufficiently high probability, we get $Y_{\tau_{1}} \geq h$. We know that the probability of $Q_{\tau_{1}}<-2 \lambda n^{-1 / 3} R^{2 / 3}$ or $\left|R_{\tau_{1}}-R\right|>R / 2$ is small by Lemmas 3.1 and 3.2. So it remains to bound $\operatorname{Pr}\left(\tau_{1}=T_{1}\right)$. For $t \leq \tau_{1}$, if $Y_{t-1}>0$, then by (1), (2), (23) and the fact that $Y_{t-1}<h$ :

$$
\mathbb{E}\left[Y_{t}^{2}-Y_{t-1}^{2} \mid C_{t-1}\right]=\mathbb{E}\left[\eta_{t}^{2} \mid C_{t-1}\right]+2 \mathbb{E}\left[\eta_{t} Y_{t-1} \mid C_{t-1}\right] \geq R_{t-1}+2 h \min \left(Q_{t-1}, 0\right) \geq \frac{R}{2}-\frac{R}{4} \geq R / 4,
$$

Also if $Y_{t-1}=0$, then by (4) we have

$$
\mathbb{E}\left[Y_{t}^{2}-Y_{t-1}^{2} \mid C_{t-1}\right]=\mathbb{E}\left[\eta_{t}^{2} \mid C_{t-1}\right] \geq R_{t-1} / 2 \geq R / 4
$$

Thus $Y_{\min \left(t, \tau_{1}\right)}^{2}-\frac{1}{4} R \min \left(t, \tau_{1}\right)$ is a submartingale, so we can apply the Optional Stopping Theorem to obtain:

$$
\mathbb{E} Y_{\tau_{1}}^{2}-\frac{R}{4} \mathbb{E} \tau_{1} \geq Y_{0}^{2} \geq 0
$$

and as $Y_{\tau_{1}} \leq 2 h$,

$$
\mathbb{E} \tau_{1} \leq \frac{4}{R} \mathbb{E} Y_{\tau_{1}}^{2} \leq \frac{16 h^{2}}{R}
$$

Hence

$$
\operatorname{Pr}\left[\tau_{1}=T_{1}\right] \leq \frac{16 h^{2}}{R T_{1}} .
$$

By the bound $\Delta \leq n^{1 / 3} R^{1 / 3} / \ln n$, we have $T_{1}+T_{2}<\frac{\zeta}{400} \frac{n}{\Delta}$. So Lemmas 3.1 and 3.2 imply that for sufficiently large $n$,

$\operatorname{Pr}\left[Y_{\tau_{1}}<h\right] \leq \operatorname{Pr}\left[\tau_{1}=T_{1}\right]+\operatorname{Pr}\left[Q_{\tau_{1}}<-2 \lambda n^{-1 / 3} R^{2 / 3}\right]+\operatorname{Pr}\left[\left|R_{\tau_{1}}-R\right|>R / 2\right] \leq \frac{16 h^{2}}{R T_{1}}+2 T_{1} n^{-10}<20 \sqrt{A}$.

This shows that with probability at least $1-20 \sqrt{A}, Y_{t}$ will reach $h$ within $T_{1}$ steps. If it does reach $h$, then the largest component must have size at least $h$, which is not as big as we require. We will next show that, with sufficiently high probability, it takes at least $T_{2}$ steps for $Y_{t}$ to return to zero, hence establishing that the component being exposed has size at least $T_{2}$, which is big enough to prove the theorem. Define

$$
\tau_{2}=\min \left\{s:\left(Y_{\tau_{1}+s}=0\right),\left(Q_{\tau_{1}+s}<-2 \lambda n^{-1 / 3} R^{2 / 3}\right),\left(\left|R_{\tau_{1}+s}-R\right|>R / 2\right), \text { or }\left(s=T_{2}\right)\right\} .
$$

We wish to show that, with sufficiently high probability, we get $\tau_{2}=T_{2}$ as this implies $Y_{\tau_{1}+T_{2}-1}>0$. We know that the probability of $Q_{\tau_{1}+\tau_{2}}<-2 \lambda n^{-1 / 3} R^{2 / 3}$ or $\left|R_{\tau_{1}+\tau_{2}}-R\right|>R / 2$ is small by Lemmas 3.1 and 3.2. So it remains to bound $\operatorname{Pr}\left[Y_{\tau_{1}+s}=0\right]$.

It will be convenient to view the random walk back to $Y_{t}=0$ as a walk from 0 to $h$ rather than from $h$ to 0 ; and it will also be convenient if that walk never drops below 0 . So we define $M_{s}=h-\min \left\{h, Y_{\tau_{1}+s}\right\}$, and thus $M_{s} \geq 0$ and $M_{s}=h$ iff $Y_{\tau_{1}+s}=0$. If $0<M_{s-1}<h$, then $M_{s-1}=h-Y_{\tau_{1}+s-1}$ and since $M_{s} \leq\left|h-Y_{\tau_{1}+s}\right|$, we have in this case that:

$$
\begin{aligned}
M_{s}^{2}-M_{s-1}^{2} & \leq\left(h-Y_{\tau_{1}+s}\right)^{2}-\left(h-Y_{\tau_{1}+s-1}\right)^{2} \\
& =2 h\left(Y_{\tau_{1}+s-1}-Y_{\tau_{1}+s}\right)+Y_{\tau_{1}+s}^{2}-Y_{\tau_{1}+s-1}^{2} \\
& =\eta_{\tau_{1}+s}\left(Y_{\tau_{1}+s}+Y_{\tau_{1}+s-1}-2 h\right) \\
& =\eta_{\tau_{1}+s}\left(\eta_{\tau_{1}+s}-2 M_{s-1}\right) \\
& =\eta_{\tau_{1}+s}^{2}-2 \eta_{\tau_{1}+s} M_{s-1} .
\end{aligned}
$$


If $M_{s-1}=0$, then $Y_{\tau_{1}+s-1} \geq h$ and so

$$
M_{s}^{2}-M_{s-1}^{2}=M_{s}^{2} \leq \eta_{\tau_{1}+s}^{2} .
$$

For $1 \leq s \leq \tau_{2}$, we have $M_{s-1}<h,(19)$ and by (4) we have $\mathbb{E}\left[\eta_{\tau_{1}+s}^{2}\right]=R_{\tau_{1}+s} \leq \frac{3}{2} R$ since $\left|R_{\tau_{1}+s}-R\right| \leq$ $R / 2$. Applying those, along with (26), (27) and (23) we conclude that such values of $s$,

$$
\begin{aligned}
\mathbb{E}\left[M_{s}^{2}-M_{s-1}^{2} \mid C_{\tau_{1}+s-1}, \tau_{1}\right] & \leq \max \left(\mathbb{E}\left[\eta_{\tau_{1}+s}^{2} \mid C_{\tau_{1}+s-1}, \tau_{1}\right], \mathbb{E}\left[\eta_{\tau_{1}+s}^{2}-2 \eta_{\tau_{1}+s} M_{s-1} \mid C_{\tau_{1}+s-1}, \tau_{1}\right]\right) \\
& \leq \max \left(\frac{3 R}{2}, \frac{3 R}{2}-2 h Q_{\tau_{1}+s-1}\right) \leq \frac{3 R}{2}+\frac{R}{4}<2 R .
\end{aligned}
$$

Let $\mathbb{E}_{h}$ and $\operatorname{Pr}_{h}$ denote respectively the conditional expectation and the conditional probability given the event $\left\{Y_{\tau_{1}} \geq h\right\}$. So $M_{s \wedge \tau_{2}}^{2}-2 R\left(s \wedge \tau_{2}\right)$ is a supermartingale under $\mathbb{E}_{h}$, and the Optional Stopping Theorem yields:

$$
\mathbb{E}_{h}\left[M_{\tau_{2}}^{2}-2 R \tau_{2}\right] \leq \mathbb{E}_{h} M_{0}^{2}=0
$$

This, along with the fact that $\tau_{2} \leq T_{2}$ yields:

$$
\mathbb{E}_{h} M_{\tau_{2}}^{2} \leq 2 R \mathbb{E}_{h} \tau_{2} \leq 2 T_{2} R
$$

Hence by (25) and Lemmas 3.1 and 3.2, we have that for $n$ sufficiently large:

$$
\begin{aligned}
& \operatorname{Pr}_{h}\left[\tau_{2}<T_{2}\right] \leq \underset{h}{\operatorname{Pr}}\left[M_{\tau_{2}} \geq h\right]+\underset{h}{\operatorname{Pr}}\left[Q_{\tau_{1}+\tau_{2}}<-2 \lambda n^{-1 / 3} R^{2 / 3}\right]+\underset{h}{\operatorname{Pr}}\left[\left|R_{\tau_{1}+\tau_{2}}-R\right|>R / 2\right] \\
& \leq \frac{\mathbb{E}_{h} M_{\tau_{2}}^{2}}{h^{2}}+\frac{2 T_{2} n^{-10}}{\operatorname{Pr}\left[Y_{\tau_{1}} \geq h\right]} \leq \frac{2 T_{2} R}{h^{2}}+\frac{2 T_{2} n^{-10}}{1-20 \sqrt{A}} \leq \frac{3 T_{2} R}{h^{2}}
\end{aligned}
$$

Combining this with (25) we conclude

$$
\operatorname{Pr}\left[\left|\mathcal{C}_{\max }\right|<T_{2}\right] \leq \operatorname{Pr}\left[\tau_{2}<T_{2}\right] \leq \operatorname{Pr}\left[Y_{\tau_{1}}<h\right]+\underset{h}{\operatorname{Pr}}\left[\tau_{2}<T_{2}\right] \leq 20 \sqrt{A}+\frac{3 T_{2} R}{h^{2}}=23 \sqrt{A}<\epsilon,
$$

for $A<\left(\frac{\epsilon}{23}\right)^{2}$. (Recall that we also require $A<(16 \lambda)^{-4}$.) This proves that Theorem 1.1(a) holds for a random configuration. Proposition 2.2 implies that it holds for a random graph.

Remark: Recall that in Section 1.2 we said that if one vertex $v$ has degree $\Delta \gg n^{1 / 3}$ and all other vertices have small degrees - small enough that the degree sequence obtained by removing $v$ has $R=O(1)$ and $Q=O\left(n^{-1 / 3}\right)$ - then with high probability there will be a component of size $O\left(n^{1 / 3}\right)$. To prove this, we follow the proof of Theorem 1.1(a), beginning the branching process with $v$. Note that this yields $R_{i}=O(1)$ for every $i \geq 0$, and this allows us to replace $R$ by $R_{0}=O(1)$ throughout the proof. Thus, eg. we set $T_{1}:=n^{2 / 3} R_{0}^{-1 / 3}, T_{2}:=A n^{2 / 3} R_{0}^{-1 / 3}$ and $h:=A^{1 / 4} n^{1 / 3} R_{0}^{1 / 3}$. Lemmas 3.1 and 3.2 are easily seen to hold with $R$ replaced by $R_{0}$. Note that (for $n$ sufficiently large) $h<\Delta=d_{v}=Y_{0}$, and so we can skip the first part of the proof, where we show that $Y_{i}$ eventually reaches $h$ with high probability.

\section{$7 \quad$ Proof of Theorem 1.3}

We close this paper with the supercritical range; i.e. when $Q>\omega(n) n^{-1 / 3} R^{2 / 3}$, where $\omega(n)$ grows with $n$. We wish to show that there exists a constant $A$ such that with probability at least $1-\epsilon$, the largest component has size at least $A Q n / R$.

The same argument as used for the proof of Theorem 1.1(a) applies here. In fact, the argument is a bit simpler here since we will always have the drift $Q_{t}>0$.

We fix $A$ later, and set $h=A^{1 / 4} \sqrt{Q n}, T_{1}=\frac{\zeta}{2000} Q n / R$ and $T_{2}=A Q n / R$. If $A<\frac{\zeta}{2000}$ then Lemmas 3.1 and 3.2 imply that for any $t \leq T_{1}+T_{2}$ we have, with high probability, $\left|R_{t}-R\right| \leq \frac{1}{2} R$ and 
$\left|Q_{t}-Q\right| \leq \frac{1}{2} Q+\frac{800}{\zeta} n^{-1 / 3} R^{2 / 3}<\frac{3}{4} Q$ for $n$ sufficiently large in terms of $\omega$. So we define our stopping times as:

$$
\begin{aligned}
\tau_{1} & =\min \left\{t:\left(Y_{t} \geq h\right),\left(Q_{t}<\frac{1}{4} Q\right),\left(\left|R_{t}-R\right|>R / 2\right), \text { or }\left(t=T_{1}\right),\right\} \\
\tau_{2} & =\min \left\{s:\left(Y_{\tau_{1}+s}=0\right),\left(Q_{\tau_{1}+s}<\frac{1}{4} Q\right),\left(\left|R_{\tau_{1}+s}-R\right|>R / 2\right), \text { or }\left(s=T_{2}\right) .\right.
\end{aligned}
$$

Note that the analogue (19) holds trivially since for $t \leq \tau_{1}+\tau_{2}$ we have $Q_{t}>0$. In fact, (19) was only required to deal with the possibility that $Q_{t}$ was negative, and so it is not needed for this case.

For $n$ sufficiently large, the same arguments (simplified slightly since $Q_{t} \geq 0$ ) still yield:

$$
\begin{aligned}
& \operatorname{Pr}\left[Y_{\tau_{1}}<h\right] \leq \frac{16 h^{2}}{R T_{1}}+2 T_{1} n^{-10}<20 \sqrt{A} \times \frac{2000}{\zeta}, \\
& \operatorname{Pr}_{h}\left[\tau_{2}<T_{2}\right] \leq \frac{3 T_{2} R}{h^{2}} \leq 3 \sqrt{A},
\end{aligned}
$$

and so $\operatorname{Pr}\left[\left|\mathcal{C}_{\max }\right|<T_{2}\right] \leq 20 \sqrt{A} \times \frac{2000}{\zeta}+3 \sqrt{A}<\epsilon$ for $A$ sufficiently small. This proves that Theorem 1.3 holds for a random configuration. Proposition 2.2 implies that it holds for a random graph.

\section{References}

[1] W. Aiello, F. Chung and L. Lu. A random graph model for massive graphs. Proceedings of the Thirty-Second Annual ACM Symposium on Theory of Computing (2000), 171-180.

[2] R. Arratia, A. Barbour and S. Tavaré. Logarithmic Combinatorial Structures: a Probabilistic Approach. European Math Society, Zurich (2003).

[3] E. Bender and R. Canfield. The asymptotic number of labelled graphs with given degree sequences. J. Comb. Th. (A) 24 (1978), 296 - 307.

[4] B. Bollobás. A probabilistic proof of an asymptotic formula for the number of labelled graphs. Europ. J. Comb. 1 (1980), 311 - 316.

[5] B. Bollobás. The evolution of random graphs. Trans. Am. Math. Soc. 286 (1984), 257 - 274.

[6] B. Bollobás. Random Graphs. 2nd Edition. Cambridge University Press (2001).

[7] F. Chung and L. Lu. Concentration inequalities and martingale inequalities: a survey. Internet Math. 3 (2006), 79 - 127.

[8] C. Cooper and A. Frieze. The size of the largest strongly connected component of a random digraph with a given degree sequence. Combin. Prob. \& Comp. 13 (2004), 319 - 338.

[9] P. Erdős and A. Rényi. On the evolution of random graphs. Magayar Tud. Akad. Mat. Kutato Int. Kozl. 5 (1960), 17 - 61.

[10] N. Fountoulakis and B. Reed. Critical conditions for the emergence of a giant component. Proceedings of Eurocomb 2009. (Journal version in preparation.)

[11] S. Jansen. The probability that a random multigraph is simple. Combin. Prob. \& Comp. 18 (2009), $205-225$.

[12] S. Jansen and M. Łuczak. A new approach to the giant component problem. Rand. Struc \& Alg. (to appear).

[13] M. Kang and T. Seierstad. The critical phase for random graphs with a given degree sequence. Combin. Prob. \& Comp 17 (2008), 67 - 86. 
[14] R. Karp. The transitive closure of a random digraph. Rand. Struc \& Alg. 1 (1990), 73 - 94.

[15] D. Levin, Y. Peres and E. Wilmer. Markov Chains and Mixing Times. American Math. Soc. (2008).

[16] T. Łuczak. Component behaviour near the critical point of the random graph process. Rand. Struc. \& Alg. 1 (1990), 287 - 310.

[17] M. Molloy and B. Reed. A critical point for random graphs with a given degree sequence. Rand. Struc. \& Alg. 6 (1995), 161 - 180.

[18] M. Molloy and B. Reed. The size of the largest component of a random graph on a fixed degree sequence. Combin. Prob. \& Comp 7 (1998), 295 - 306.

[19] A. Nachmias and Y. Peres. The critical random graph with martingales. Israel J. Math. (to appear).

[20] A. Nachmias and Y. Peres. Component sizes of the random graph outside the scaling window. Latin Am. J. Prob. and Math. Stat. 3 (2007), 133 - 142.

[21] M. Newman, A. Barabási and D. Watts. The Structure and Dynamics of Networks. Princeton University Press (2006). 